\title{
Forecasting Risk of Crop Disease with Anomaly Detection Algorithms
}

\author{
Peter Skelsey ${ }^{\dagger}$ \\ Information and Computational Sciences, James Hutton Institute, Dundee, United Kingdom \\ Accepted for publication 31 July 2020.
}

ABSTRACT

\begin{abstract}
Information from crop disease surveillance programs and outbreak investigations provides real-world data about the drivers of epidemics. In many cases, however, only information on outbreaks is collected and data from surrounding healthy crops are omitted. Use of such data to develop models that can forecast risk/no risk of disease is therefore problematic, as information relating to the no-risk status of healthy crops is missing. This study explored a novel application of anomaly detection techniques to derive models for forecasting risk of crop disease from data composed of outbreaks only. This was done in two steps. In the training phase, the algorithms were used to learn the envelope of weather conditions most associated with historic crop disease outbreaks. In the testing phase, the algorithms were used for hindcasting of historic outbreak events. Five different anomaly detection algorithms were compared according to their accuracy in
\end{abstract}

forecasting outbreaks: robust covariance, one-class $k$-means, Gaussian mixture model, kernel density estimation, and one-class support vector machine. A case study of potato late blight survey data from across Great Britain was used for proof of concept. The results showed that Gaussian mixture model had the highest forecast accuracy at $97.0 \%$, followed by one-class $k$-means at $96.9 \%$. There was added value in combining the algorithms in an ensemble to provide a more accurate and robust forecasting tool that can be tailored to produce regionspecific alerts. The techniques used here can easily be applied to outbreak data from other crop pathosystems to derive tools for agricultural decision support.

Keywords: anomaly detection, crop, decision support, disease, disease control, forecast, machine learning, model, pest management, risk
Knowledge and information are key for correct pest management decisions. Integrated pest management (IPM) is a system that emphasizes appropriate decision making, which depends heavily on intensive, accurate, and timely information for field implementation by practitioners (Arora et al. 2012). Disease forecasting is an important component of the broad IPM philosophy, as forecasting models provide a lead time for managing impending outbreaks and thus minimize crop loss, chemical inputs, and production costs and enhance safeguards to the environment and human health.

Models for forecasting risk of crop disease are typically derived from experiments in the laboratory, glasshouse, or controlled environment chambers or from statistical relationships between environmental data and disease observations in small-plot field trials or trap plants. An argument can be made, however, that experiments conducted under highly standardized conditions or at a small spatial or temporal scale can produce results with little validity beyond the specific environment in which the experiment is conducted. Plant disease epidemics occur at the landscape scale and are influenced by aspects of the wider environment, such as topography, climate, and variability in host and pathogen populations (Skelsey et al. 2010). Landscape-scale experiments to derive and test models of disease risk are rarely conducted, but surveillance programs for crop diseases and outbreak investigations can provide useful real-world data about the drivers of disease at the spatial scale of interest.

\section{†Corresponding author: P. Skelsey; peter.skelsey@hutton.ac.uk}

Funding: This research was supported by the Rural and Environment Science and Analytical Services (RESAS) Division of the Scottish Government under its Environmental Change and Food, Land and People Strategic Research Programmes (2016 to 2021).

*The $\boldsymbol{e}$-Xtra logo stands for "electronic extra" and indicates that two supplementary files are published online.

The author(s) declare no conflict of interest.

C 2021 The American Phytopathological Society
Machine learning (ML) has emerged together with big data technologies and high-performance computing to create new opportunities to unravel, quantify, and understand data-intensive processes in agricultural environments (Liakos et al. 2018). ML can be defined as a subset of artificial intelligence that provides specialized algorithms that can learn from data automatically and improve from experience, without being explicitly programmed to do so (Samuel 1959). Most applications of ML in agriculture have focused on image processing tasks, such as weed detection and identification (Ishak et al. 2009; Rahman et al. 2015), detection of disease symptoms (Ferentinos 2018; Ghosal et al. 2018; Huang et al. 2015; Ma et al. 2015; Mutka and Bart 2015; Sladojevic et al. 2016; Yamamoto et al. 2017), pest identification (Boniecki et al. 2015; Silva et al. 2015; Venugoban and Ramanan 2014), vegetation area detection (Guo et al. 2013), flower detection (Guo et al. 2015), leaf counting (Ubbens et al. 2018), and fruit detection (Kurtulmus et al. 2014; Linker et al. 2012; Rahnemoonfar and Sheppard 2017; $\mathrm{Sa}$ et al. 2016; Yamamoto et al. 2014). Another important application of ML in agriculture is that of yield prediction, one of the most significant topics in precision agriculture, with examples for beans, corn, potatoes, and tomatoes (Gonzalez-Sanchez et al. 2014) as well as rice (Delerce et al. 2016; Su et al. 2017) and maize (Marko et al. 2017). ML excels at this task because of its ability to discover complex relationships between a variety of factors and filter out unimportant factors. It is therefore surprising that examples of ML to forecast risk of crop disease are sporadic in the literature. A few pioneering articles include the application of ML techniques to forecast risk of wheat tan spot (De Wolf and Franel 1997), rice blast (Kaundal et al. 2006), wheat stripe rust (Chen et al. 2006), Fusarium head blight (Landschoot et al. 2013), wheat yellow rust (Xu et al. 2018), and powdery mildew (Hamer et al. 2020). Despite the predictive success of the ML algorithms developed in these studies, there is still an overwhelming reliance on traditional statistical procedures and mechanistic modeling approaches for crop disease prediction in plant disease epidemiology.

Many decision support systems (DSSs) for crop protection are used to make binary decisions about whether to apply a crop protectant or other control measure (De Wolf and Isard 2007). This 
approach can be framed as a ML classification problem, where input data are used to discriminate between healthy/diseased crops and derive a model to predict risk/no risk of disease. In a conventional classification problem, data from each class of interest are available (e.g., healthy and diseased crops), and each object is represented by a vector of features (e.g., date, location, weather data, crop characteristics). Training an ML algorithm involves feeding training examples from both classes to the algorithm so that it can create rules to assign membership based on the individual feature values. When encountering new instances, they can be classified according to the learned rules. The one-class classification problem differs in one essential aspect from the conventional classification problem: only information of one of the classes, the target class, is available (Désir et al. 2013; Khan and Madden 2010; Mazhelis 2006; Tax 2001). This means that just example objects of the target class can be used for training the classifier and that no information about the other class of objects is present. This is an interesting problem because this is typically the case for surveillance programs for crop diseases or outbreak investigation data, where only outbreaks are recorded and data from surrounding healthy crops are omitted. Although such data are abundant in botanical epidemiology and undoubtedly contain useful information on the drivers of disease, they are seldom used for the development of forecasting tools because of the absence of information on the disease-free or risk-free status. The task, then, is to explore these data to find the feature values (e.g., weather variables) most commonly associated with disease occurrence and those that are less common or anomalous, in order to derive rules that can be used to predict risk of disease (or no risk) in the future. One-class classification is therefore often referred to as outlier or anomaly detection.

It is often difficult to construct a single "best" model for forecasting risk of crop disease based on environmental variables (Hu et al. 2015). Effective results can often be obtained on some parts of the data that are modeled well (e.g., certain years), whereas results on other parts of the data may not be accurate. Similarly, a given model may be accurate on the dataset used for learning but may not generalize well to unseen data (e.g., from a different geographic region) (Aggarwal and Sathe 2018). In the anomaly detection problem, there exists a multitude of approaches, including nearest neighbor-, distance-, density-, cluster-, and classificationbased ML methods (Kaur and Singh 2016). Within these approaches, there exists a myriad of algorithms that are designed to solve the same problem. With such an array of methods for prediction, many of which could have similar levels of predictive accuracy, the question is how to select the best one. Unfortunately, the modeling process is often inherently subjective, where the model developed for a particular problem depends on the analyst's understanding of the data. An obvious approach to overcome this problem is to rigorously compare alternative models and test them on data not used in the learning process. A less common approach, at least in botanical epidemiology, is to combine models for prediction into ensembles. Ensemble analysis is a method commonly used in data mining and ML to reduce the dependence of the model on the specific data and greatly increase the robustness of predictions (Benkeser et al. 2018; Krawczyk et al. 2017; Luo et al. 2018). In the context of the classification problem, a variety of ensemble-based methods have been proposed to combine the predictions of independent or dependent models, such as bagging (Brieman 1996), boosting (Freund and Schapire 1997), stacking (Clarke 2004), model averaging (Domingos 2000), and bucket of models (Madasamy and Ramaswami 2017). The idea is to confront a prediction problem with different types of models, each of which can learn a part of the problem space, not the whole problem space. This is based on the "no free lunch theorem" of Wolpert (2002), which states, "There is not a single classifier that is appropriate for all the tasks, since each algorithm has its own domain of competence."
In this study, a range of different anomaly detection algorithms were applied to potato late blight (PLB) survey data from across Great Britain to learn the "normal" weather conditions most associated with outbreaks of disease and derive models for forecasting risk of disease. The predictive power of each individual algorithm as a tool to forecast outbreaks is reported as well as the results from the algorithms combined in a multimodel ensemble. Two further models were included as a baseline for comparison with the anomaly detection algorithms: the Smith Period (SP) was developed in the 1950s as a DSS to indicate high-risk periods for PLB development in Great Britain (Smith 1956); this was replaced by the Hutton Criteria (HC) in 2017, which is the current national warning system for late blight in Great Britain via the online service Blightwatch (https://blightwatch.co.uk/). To my knowledge, this is the first successful research work on applying anomaly detection techniques to the task of crop disease forecasting. A desktop app that enables the user to train and test their own crop disease forecasting tool using the presented methodologies is provided. The source code is the core of a future commercial application; therefore, the contents of the app are encrypted to protect intellectual property rights, but it is freely available to download and use via the link in Supplementary File S1.

\section{MATERIALS AND METHODS}

Epidemic data. The late blight outbreak data spanned a 6-year period (2012 to 2017) and consisted of the date, coordinates (U.K. postcode district centroid), genotype, and stage of outbreak of 1,049 late blight outbreaks from across Great Britain (Fig. 1). These data are collected routinely each year as part of the Agriculture and Horticulture Development Board (AHDB) Potatoes Fight Against Blight (FAB) service (https://potatoes.ahdb.org.uk/online-toolbox/ fight-against-blight-tool). Outbreaks are reported on a voluntary basis by members of the potato industry (e.g., agronomists) who regularly walk potato fields during the season. Samples of suspected plant material are collected and sent for analysis to provide information to the potato industry on blight incidence and pathogen population structure. The outbreak data are subject to a data sharing agreement between the author and AHDB Potatoes and under these terms may not be made publicly available.

Historical hourly weather data corresponding to every outbreak location were provided by the U.K. Meteorological Office (UKMO). The data were interpolated by UKMO from their network of weather stations to provide values for 9,000 postcode sectors, which were aggregated to the postcode district level to conform to the outbreak data. The weather data are subject to a data sharing agreement between the author and UKMO and under these terms may not be made publicly available, but they can be purchased directly from UKMO. To gain insight on the accuracy and performance of the algorithms, two weather data subsets were created. The first was composed of weather data spanning a 28-day period prior to the date that each outbreak was reported. This was considered appropriate for relating weather conditions for infection to the dates at which disease was observed in the crop, as the majority $(71.4 \%)$ of reported outbreaks where stage of outbreak was recorded were described as "multiple patches/severe/widespread/ scattered," suggesting that multiple infection cycles had occurred before the outbreak was sampled and recorded. On each day in each 28-day window, the minimum daily temperature and the total number of hours each day of RH $\geq 90 \%$ were calculated, where $90 \%$ $\mathrm{RH}$ is a proxy for leaf wetness (Smith 1956). These two variables were selected because they are historically used to calculate late blight risk alerts in Great Britain: the SP and the HC (described below). These data were used as input features (predictor variables) to train and test the algorithms for their accuracy in forecasting outbreaks $(1,045$ outbreaks $\times 28$ days $=29,260$ values of the two predictor variables). A second important consideration for a crop disease forecasting system is the frequency of alerts issued 
throughout the growing season, as too many alerts can overwhelm the user and result in a system that is of little practical use. To assess the seasonal frequency of alerts, values of daily minimum temperature and number of hours each day of $\mathrm{RH} \geq 90 \%$ spanning the Great Britain potato growing season (1 May to 31 October) were compiled for each outbreak (1,045 outbreaks $\times 184$ days $=192,280$ values of the two predictor variables).

Statistical methods. Among the many anomaly detection algorithms available, five of the most commonly used were selected to compare for forecasting outbreaks (Goldstein and Uchida 2016). Each algorithm assigns an anomaly score to each vector of the two weather-based predictor variables, describing its outlier tendency. The scores are converted to a binary prediction ( 0 or 1$)$ by imposing a user-defined decision threshold to create a decision boundary separating "normal" samples from anomalies. In the current context, the decision boundary is used to define the envelope of weather conditions most associated with outbreaks (i.e., the least anomalous data). Weather conditions that fall inside the boundary trigger a disease risk alert, otherwise no alert is issued. As the decision threshold value determines the size and shape of the decision boundary, it can be used to control the frequency of alerts issued. A brief description of each algorithm is given, including a multimodel ensemble of all algorithms, with references provided for additional details. The MATLAB commands used to implement the algorithms are given below, and pseudocode is provided for the techniques used to train, tune, and test the algorithms and develop "finalized" model versions for operational use (Supplementary File S2).

Robust covariance. Distance-based techniques identify outliers as data points that are situated away from the majority of points using a geometric distance measure. One of the commonly used distance metrics in the multivariate space is the Mahalanobis distance (MD) (Mahalanobis 1936), which considers the mean and covariance of the data, and for which larger distances are returned for observations that deviate from the mean in directions with smaller covariance (Aggarwal 2013). This curbs the problem of scale and correlation that is inherent to Euclidian distance. Equal MD contours appear as ellipses rotated according to the correlation of the variables, and any point whose MD from the center of mass of the data is greater than a user-defined threshold is classed as an anomaly. Nevertheless, both the arithmetic mean and the sample covariance matrix are highly sensitive to outlying observations and using these estimators for multivariate outlier detection can lead to questionable results (Maronna et al. 2006). Robust distance methods attempt to assess whether an observation is outlying using the MD; however, rather than using the classical mean and covariance estimate for the data when computing the distance, robust estimates are used instead. The minimum covariance

\section{A}

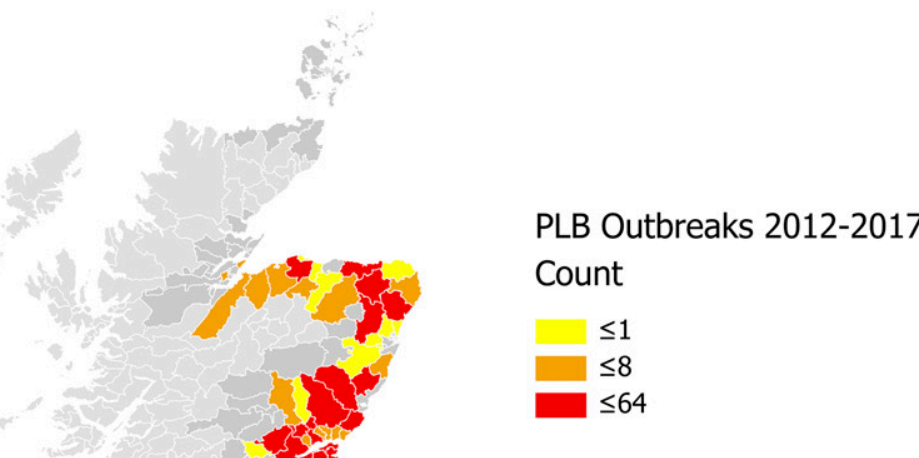

Base layers

$\geq 1$ ha potato

Postcode districts

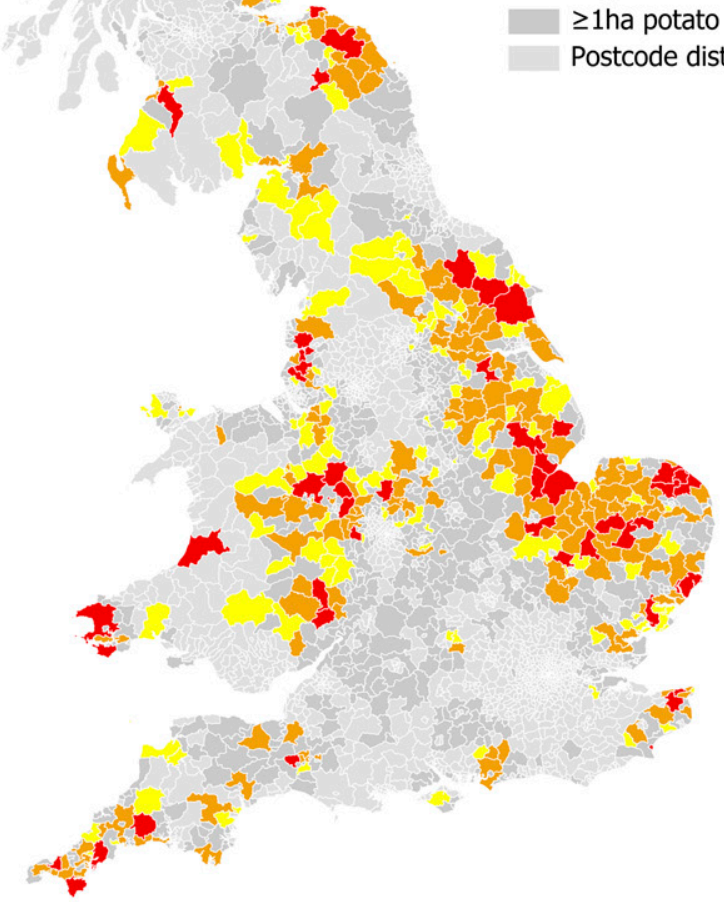

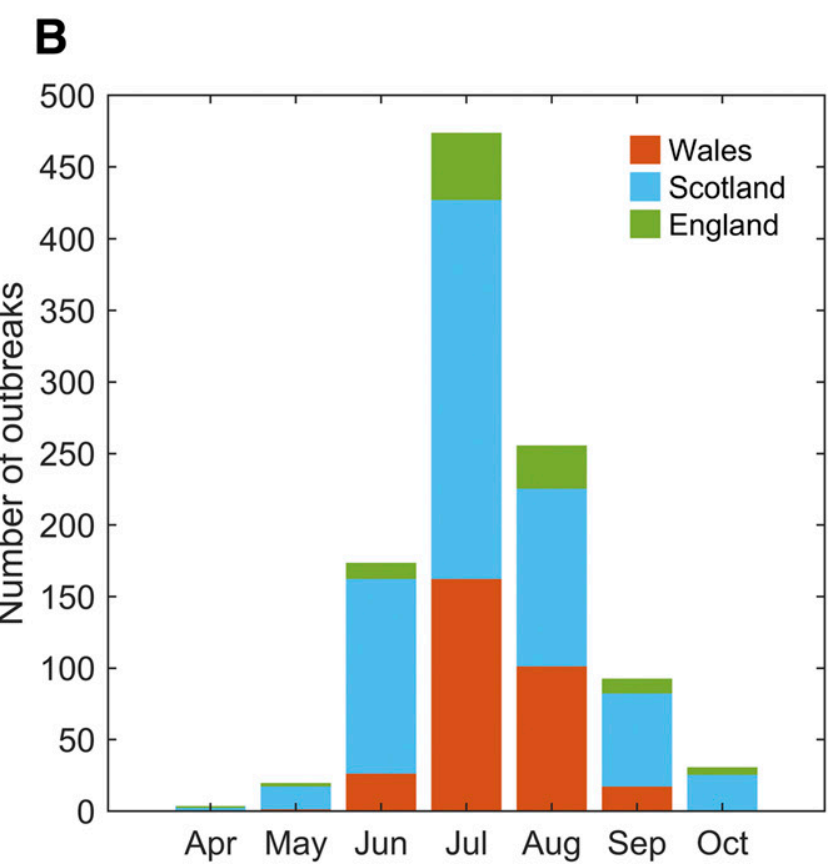

Fig. 1. A, Distribution of potato late blight (PLB) incidence data used for model development and testing. B, Cases of late blight by month of onset (date of reporting), grouped by country. 
determinant (MCD) estimator is one of the first affine equivariant and highly robust estimators of multivariate location and covariance (Rousseeuw 1984, 1985). Being resistant to outlying observations makes the MCD very useful for anomaly detection. Although MCD was introduced in 1984, it has become increasingly popular since the construction of the computationally efficient Fast-MCD algorithm of Rousseeuw and Van Driessen (1999). In this study, robust covariance (RCOV) was determined by calculating robust distances using Fast-MCD via the MATLAB procedure ROBUSTCOV, with default settings.

One-class k-means. The $k$-means classifier is based on the wellknown $k$-means clustering algorithm (Jain 2010; Jain and Dubes 1988; Lloyd 1982). The algorithm identifies $k$ clusters in the data by iteratively assigning each observation to the nearest cluster. The algorithm can be used for anomaly detection by clustering the training set and then computing the Euclidean distance in feature space of a test observation to the cluster centroids. If the distance is larger than a user-defined decision threshold, the observation will be classified as an anomaly. As the result depends on the initial centroids selection, this process was repeated 10 times and the best initial centroids were selected. One-class $k$-means (OCKM) was implemented using the MATLAB procedure KMEANS with replicates set to 10 , default settings elsewhere, and tuned for the hyperparameter $k$ (number of clusters). The method for tuning is defined in the section on nested $k$-fold cross-validation and model selection.

Gaussian mixture model. Density-based techniques take the local density into account when searching for outliers. These techniques can effectively identify local outliers in datasets with diverse clusters. A mixture model is a probabilistic model for representing the presence of subpopulations (clusters) within an overall population, without requiring that an observed data set should identify the cluster to which an individual observation belongs (Lindsay 1995; Rocci et al. 2018). Whereas the $k$-means algorithm performs a "hard assignment" of data points to clusters, in which each data point is associated uniquely with one cluster, mixture models make a "soft assignment," where each data point can belong to any cluster with a given probability. In a Gaussian mixture model (GMM), each cluster is approximated by a Gaussian distribution characterized by a mean as the center of the cluster and a variance. Formally, a GMM is a parametric probability density function represented as a weighted sum of these Gaussian component densities (Lindsay 1995). A GMM is therefore parameterized by two types of values: the mixture component weights, and the component means and variances/covariances. If the probability assigned to a datum is less than the user-defined decision threshold, then it is classed as an anomaly. GMM was implemented using the MATLAB procedure FITGMDIST with Regularization Value set to 0.01 , replicates set to 10 , default settings elsewhere, and tuned for the hyperparameter $k$ (number of Gaussian components).

Kernel density estimation. Kernel density estimation (KDE) is a well-established nonparametric method of estimating the probability density function (PDF) of a finite dataset (Silverman 2018). It is nonparametric because unlike GMM it does not assume any underlying distribution for the data. KDE calculates the density of events around each observation by assigning a kernel function to every datum that weights the distances to other points in the feature space. Several kernel functions have been proposed, of which the most common are the linear, Gaussian, box, triangle, and epanechnikov kernels (Soh et al. 2013). Each kernel has a bandwidth (smoothing) parameter that controls the size of the neighborhood around each datum. The PDF is then produced by summing the local contributions of the kernels and dividing by the number of observations to ensure that it satisfies the required properties of a PDF. If the probability assigned to a datum is less than a user-defined decision threshold, then it is classed as an anomaly. KDE was implemented using the MATLAB procedure KSDENSITY with default settings.
One-class support vector machine. The one-class support vector machine (OCSVM) is one of the most popular and successful algorithms for anomaly and novelty detection (Schölkopf and Smola 2001; Schölkopf et al. 2001). The algorithm implements the following idea: it maps the input data into a high-dimensional feature space and constructs an optimal separating hyperplane that maximizes the margin, which is the distance between the hyperplane and the nearest data points (support vectors) of each class (normal or abnormal) of data in the space (Schölkopf and Smola 2001; Schölkopf et al. 2001; Vapnik 1995). This means that data points that cannot be separated by a straight line in their original space (e.g., two-dimensional) are "lifted" to a feature space (e.g., three-dimensional) where there can be a "straight" hyperplane that separates the desired fraction of anomalies from the input data. When that hyperplane is projected back to the original input space, it has the form of a nonlinear curve. Different mappings construct different SVMs. The mapping is performed by a kernel function, the most common being the following: linear, radial basis function, polynomial, and sigmoid (Hsu et al. 2001). The OCSVM then makes predictions by assigning new points to one side of the hyperplane or the other, returning a positive score for the normal class and a negative score for the abnormal class. OCSVM was implemented using the MATLAB procedure FITCSVM with a radial basis function kernel, OutlierFraction set to 6/7, default settings for one-class learning elsewhere, and tuned for the hyperparameter KernelScale, which determines the width of the kernel.

Ensemble of anomaly detectors. For the ensemble of anomaly detectors (ENS), the five anomaly detection algorithms described above were combined in a multimodel ensemble using a "presidential voting" scheme, whereby the algorithm with the highest predictive accuracy determined the ensemble result (Knauer et al. 2019). This simple but extreme "winner-takes-all" voting method is designed to avoid the decisions of poorly performing classifiers in situations where performance can vary, such as between different time periods or geographic regions ( $\mathrm{Su}$ et al. 2018).

Preprocessing. ML algorithms that exploit distances or similarities between data samples can be sensitive to the scale and variance of input features; therefore, some form of scaling or normalization is a common data preprocessing step. This was not of great concern in the current study because the two weather features (minimum daily temperature and the total number of hours of $\mathrm{RH} \geq$ $90 \%$ ) have a similar range and variance. Nevertheless, there were potential implications for OCKM and OCSVM; RCOV uses robust estimates of the unitless and scale-invariant MD to measure multivariate distance (and thus similarities) between input features, whereas the optimization procedures in GMM and KDE are able to learn the variances of input features. Two widely used data scaling algorithms were applied as a preprocessing step for OCKM and OCSVM: the minimum-maximum algorithm was used to normalize each input variable to an interval of [0,1], and the Z-score algorithm was used to standardize each variable to a mean of 0 and a standard deviation of 1 (Lantz 2015). Subsequent to model training, tuning, and testing (see section below), both techniques were found to have a slightly detrimental effect on OCSVM, but Z-score standardization improved the accuracy of OCKM. Results are therefore provided with standardized data for OCKM and raw (untransformed) data for the other algorithms.

Nested $\boldsymbol{k}$-fold cross-validation and model selection. Each algorithm contains parameters that are learned from the data and "hyperparameters" whose value must be set before the learning process begins. In order to fairly evaluate the performance of the methods and not introduce biases in the choices of hyperparameters, a nested cross-validation scheme was implemented. Based on other studies, this procedure was selected to strictly control the information leak between the training and test data and to get an unbiased estimate of generalization accuracy to unseen data (Douglas et al. 2013; Japkowicz and Shah 2011; Krstajic et al. 
2014; Misaki et al. 2010; Varma and Simon 2006). The procedure consisted of two nested cross-validation loops: an outer one to test generalization accuracy, and an inner one for optimization of hyperparameters (also called tuning or model selection). Pseudocode is provided to facilitate implementation of the nested $k$-fold procedure (Supplementary File S2). The subset of weather data spanning a 28-day period prior to the date that each outbreak was reported was used for this analysis.

The procedure was initiated by partitioning the data into 10 nonoverlapping subsets (outer folds). Following a standard $k$-fold cross-validation approach, each fold was used once as the testing set and the remaining $k-1$ folds ( $90 \%$ of the data) as the training set. At each iteration of the outer 10-fold cross-validation loop, the training set was partitioned into 10 folds to be used in an inner 10-fold crossvalidation loop, where hyperparameter tuning was performed using a grid search over candidate values. The hyperparameter settings that gave the best cross-validated accuracy in the inner loop were selected, and the associated model was then retrained on the outer training set and evaluated on its predictive accuracy for the held-out test fold in the outer loop. This process was repeated 10 times so that each of the original 10 folds was used as the test set once and predictions were obtained for each entry in data set. Within each loop, training data were minimum to maximum or Z-score scaled
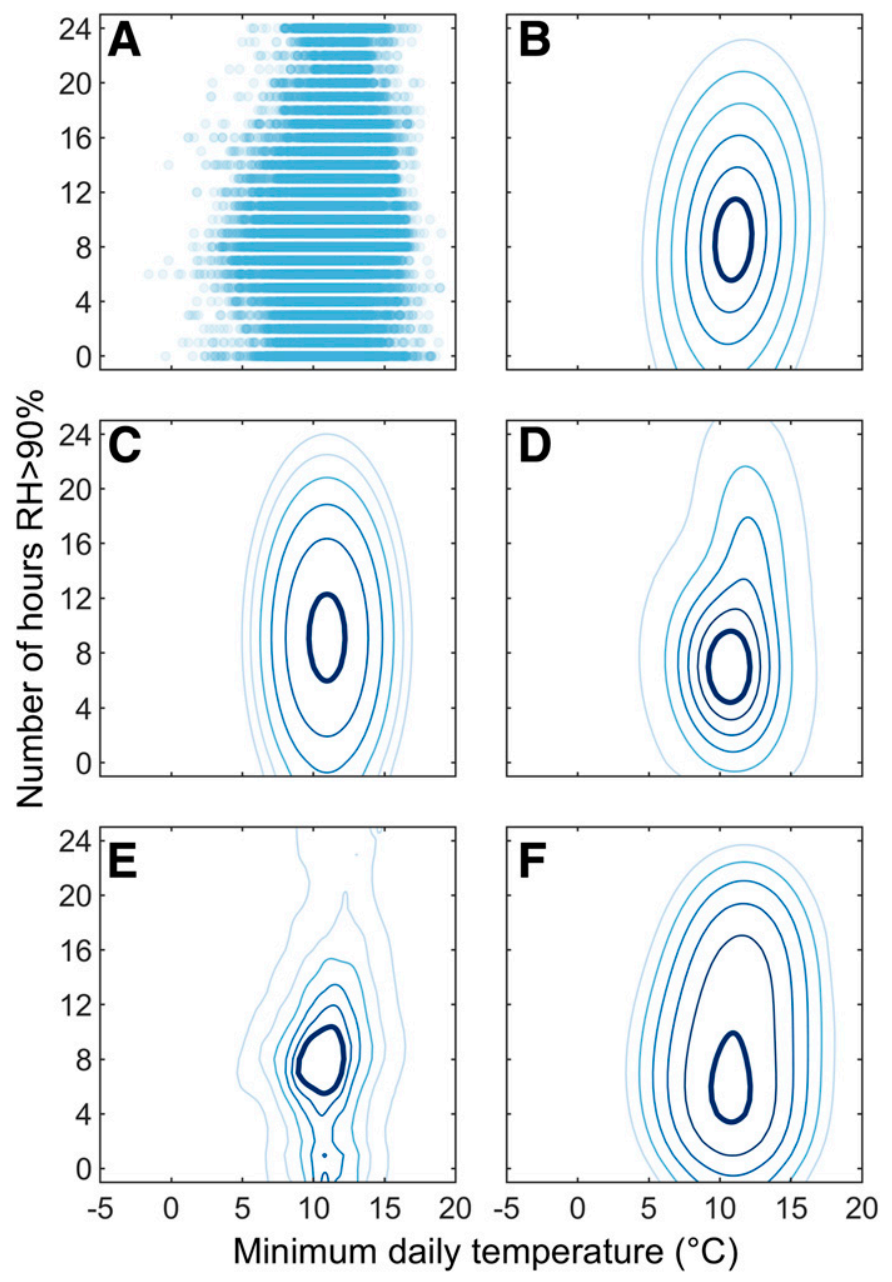

Fig. 2. Comparison of decision boundaries among five "finalized" anomaly detection algorithms retrained on the complete potato late blight outbreak data set. A, Raw weather data in the 28 days preceding each outbreak. B, robust covariance. $\mathbf{C}$, one-class $k$-means, with $k=1$. D, Gaussian mixture model, with $k=2$. E, Kernel density estimation. F, One-class support vector machine, with KernelScale $=7.5$. Lighter contours correspond to greater outlier tendency, with decision boundaries delineated in bold. Decision threshold values were set to the 14.29 percentile of anomaly scores. where appropriate, and test data were scaled using the minimum/ maximum values or mean and standard deviation of training data features.

Cross-validation accuracy was assessed in both the inner and outer loops using an extrinsic performance function, where the models were used to forecast outbreaks in the held-out test data. Any risk alert triggered in the 28-day window of weather data preceding each outbreak was classed as a "successful" forecast. The decision threshold used to separate nominal data (producing a risk alert) from anomalies was set to the 14.29 percentile of the anomaly scores for the algorithm, in order to obtain an alert frequency of one in every 7 days during the favorable disease conditions of the 28-day window preceding each outbreak. The exception here was OCSVM, in which the user can set the desired fraction of anomalies as a hyperparameter value and the decision threshold value is 0 . This choice for the decision threshold was arbitrary and based primarily on the frequency of fungicide applications for late blight control in a typical calendar spray regime in the United Kingdom. Note that the decision threshold can be tuned to obtain any alert frequency desired, and that the frequency of alerts issued over the course of an entire potato growing season (see the section on additional performance measures) is expected to be lower, providing potential to reduce the number of sprays compared with a typical calendar spray regime. Cross-validation accuracy in both loops was measured as the percentage of outbreaks successfully forecasted, averaged across the test folds. The frequency of alerts was also computed in both loops as the percentage of days in the 28-day window of test cases that received an alert, averaged across the test folds. Results for the multimodel ensemble, ENS, were determined by the most accurate algorithm per test fold.

Baseline models (SP and HC). Both the "legacy" (SP) and current $(\mathrm{HC})$ national warning systems for late blight in Great Britain are composed of simple temperature and humidity criteria that are used to forecast favorable conditions for development of late blight. The SP is defined as two consecutive days with a minimum temperature of $10^{\circ} \mathrm{C}$ and at least $11 \mathrm{~h}$ of $\mathrm{RH} \geq 90 \%$ on each day (Smith 1956). The HC modified the SP rule regarding $11 \mathrm{~h}$ of high humidity on each day, decreasing the duration of high humidity required to $6 \mathrm{~h}$ of $\mathrm{RH} \geq 90 \%$ per day, to reflect the ability of the contemporary pathogen population to infect under shorter periods of wet conditions (https://blightwatch.co.uk/). These models did not require training or tuning of hyperparameters and were tested for accuracy and frequency of alerts (where two consecutive days of favorable conditions constitute one alert) during the 28-day period preceding each outbreak using the same held-out test sets as the anomaly detection algorithms.

TABLE 1. Nested 10-fold cross-validation performance measures

\begin{tabular}{lcc}
\hline Measure $^{\mathrm{a}}$ & Accuracy $^{\mathrm{b}}$ & Frequency $^{\mathrm{c}}$ \\
\hline SP & $84.7(3.6)$ & $12.9(0.9)$ \\
HC & $97.2(1.1)$ & $31.8(2.3)$ \\
RCOV & $96.7(1.4)$ & $13.9(1.0)$ \\
OCKM & $96.9(1.9)$ & $14.3(1.3)$ \\
GMM & $97.0(1.6)$ & $14.4(0.8)$ \\
KDE & $96.0(1.7)$ & $14.3(0.9)$ \\
OCSVM & $96.8(1.9)$ & $14.3(0.9)$ \\
ENS & $98.2(0.7)$ & $14.5(1.2)$ \\
\hline
\end{tabular}

a $\mathrm{SP}=$ Smith Period, $\mathrm{HC}=$ Hutton Criteria, $\mathrm{RCOV}=$ robust covariance, OCKM $=$ one-class $k$-means, $\mathrm{GMM}=$ Gaussian mixture model, $\mathrm{KDE}=$ kernel density estimation, OCSVM = one-class support vector machine, and ENS $=$ ensemble of the five anomaly detection algorithms.

b Mean percentage accuracy in forecasting outbreaks, where any risk alert triggered in the 28-day window of weather data preceding each outbreak is classed as a "successful" forecast. Standard deviations are given in parentheses.

${ }^{c}$ Mean percentage of days on which an alert was issued. Standard deviations are given in parentheses. 
Additional performance measures. Predictions on the test folds were aggregated according to the month the outbreak was recorded to investigate temporal variation in forecast accuracy for the anomaly detection algorithms, the multimodel ensemble (ENS), and the baseline models. Results for each month-model combination were averaged across the test folds.

Temporal variation in model output was further investigated using the classifier output difference (COD) metric to determine pairwise similarity in the time series of alert predictions (Peterson and Martinez 2005). The COD was calculated as the fraction of days in a test set where the predictions (no alert or alert, represented as 0 or 1 ) did not match, giving a diversity measure between 0 and 1 for each pair of models. The mean COD across all test folds is provided for each pair of algorithms.

Predictions on the test folds were aggregated according to UKMO administrative regions to investigate geographic variation in forecast accuracy for the anomaly detection algorithms, the multimodel ensemble (ENS), and the baseline models. For this analysis, the algorithm with the highest predictive accuracy per region within each test fold determined the ENS result for that region within that test fold. Results for each region-model combination were averaged across the test folds.

To make predictions on new data, the anomaly detection algorithms were "finalized" by training on all available data. This involved an additional round of $k$-fold cross-validation for tuning of hyperparameters, followed by retraining of optimized versions using the entire 28-day weather data subset. Pseudocode is provided to clarify the process of finalizing a model for operational use (Supplementary File S2). The finalized models were then applied to the weather data subset spanning 1 May to 31 October for each outbreak (see the section on epidemic data) to calculate the frequency of alerts issued during the whole growing season. The two baseline models, SP and HC, were also applied to these data for comparison; note that these models require two consecutive days of favorable conditions to constitute a "full alert." Results were aggregated according to UKMO administrative regions to investigate geographic variation in seasonal alert frequencies. The multimodel ENS results were determined by the anomaly detection algorithm with the highest predictive accuracy per region from the previous analysis.

\section{RESULTS}

All five finalized anomaly detection algorithms produced similar decision boundaries in the temperature and $\mathrm{RH}$ data, with slight differences in the range of values classified as normal (i.e., risky for late blight development) (Fig. 2). The daily minimum temperature values defining the decision boundaries ranged from 9.7 to $12.3,9.7$ to $12.2,9.2$ to $12.1,8.9$ to 12.1 , and 9.4 to $12.1^{\circ} \mathrm{C}$ for RCOV, OCKM, GMM, KDE, and OCSVM, respectively. The number of hours of $\mathrm{RH} \geq 90 \%$ defining the decision boundaries ranged from 5.6 to 11.5 , 6.0 to $12.3,4.4$ to $9.6,5.5$ to 10.4 , and 3.4 to 9.9 for RCOV, OCKM, GMM, KDE, and OCSVM, respectively.

The current national warning system for PLB (HC) had a higher cross-validation accuracy in forecasting PLB outbreaks than the legacy model it replaced (SP), but at the expense of a much higher alert frequency during the 28-day period preceding each outbreak (Table 1). SP resulted in approximately one alert in every 8 days, and $\mathrm{HC}$ resulted in approximately one alert in every 3 days. Predictive accuracy was very high for all five anomaly detection algorithms, and alert frequency was consistent at one alert in every 7 days. The multimodel ensemble, ENS, outperformed all

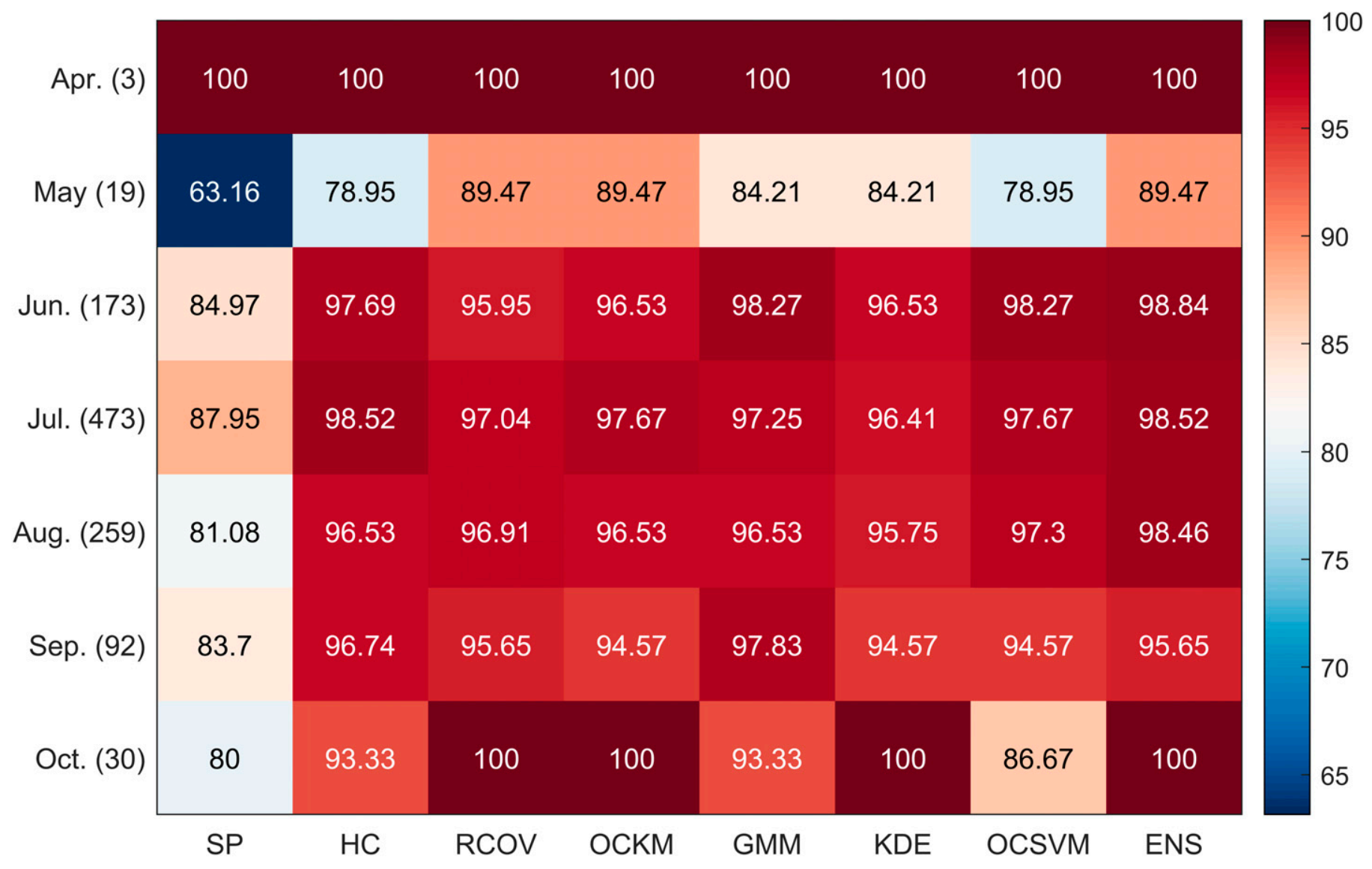

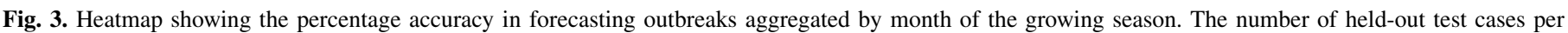

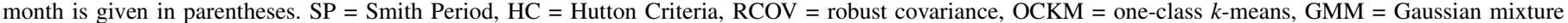
model, $\mathrm{KDE}=$ kernel density estimation, OCSVM = one-class support vector machine, and ENS = ensemble of the five anomaly detection algorithms. 
individual component algorithms, with an accuracy exceeding that of the current national warning system with less than half the number of alerts issued.

Predictive accuracy varied among the months of the growing season by $36.8,21.1,10.5,10.5,15.8,15.8,21.1$, and $10.5 \%$ for SP, HC, RCOV, OCKM, GMM, KDE, OCSVM, and ENS, respectively (Fig. 3). Predictive performance tended to be higher during the summer months for all models than for the beginning (April to May) and end (September to October) of the growing season.

The relatively higher alert frequency of HC compared with SP meant that the time series of risk alerts produced by the two baseline models were quite dissimilar (large pairwise COD values) (Fig. 4). In contrast, the time series of risk alerts produced by the anomaly detection algorithms were very similar to each other (low pairwise COD). There was little synchrony between the predictions of each baseline model and the various anomaly detection algorithms. Although the patterns of predictions produced by the anomaly detection algorithms were very similar, intermittent disparities in risk forecasts highlighted the advantage of a multimodel approach and the ensemble (ENS) served to increase confidence in the robustness of risk predictions (Fig. 5)

The accuracy of the current national warning system for late blight (HC) was markedly higher and more uniform across the different administrative regions of Great Britain than the legacy model it replaced (SP) (Fig. 6). The most accurate anomaly detection algorithm varied from region to region, with the multimodel ensemble ENS matching or outperforming $\mathrm{HC}$ in terms of predictive accuracy in all Great Britain administrative regions except East Scotland. Note that the results for London were from reported outbreaks in city allotments as opposed to commercial crops.

Seasonal alert frequencies (1 May to 31 October) of all models were consistent across Great Britain administrative regions (Fig. 7). Mean seasonal alert frequencies across all regions were 6.8, 18.5, $12.5,8.2,8.6,8.7,8.7$, and $8.3 \%$ for SP, HC, RCOV, OCKM, GMM, KDE, OCSVM, and ENS, respectively.

\section{DISCUSSION}

This work addresses the challenge of deriving models to forecast risk of crop disease from datasets composed of disease outbreaks only; in the conventional approach, predictive systems are developed with multiclass data composed of healthy (e.g., controls) and diseased cases. An anomaly detection framework was proposed, where the scoring ability of the algorithms was exploited to rank vectors of predictor variables according to their outlier tendency. A decision threshold was applied to the anomaly scores to control the frequency of alerts issued. In a case study on PLB, the resulting algorithms achieved a comparable or improved forecast accuracy with a much lower frequency of alerts compared with the current national warning system for PLB in Great Britain.

Anomaly detection is quickly becoming a very significant ML tool in a variety of domains, such as intrusion detection, fraud detection, fault detection, system health monitoring, event detection in sensor networks, and novelty detection in online settings, and is often used in preprocessing to remove outliers from data

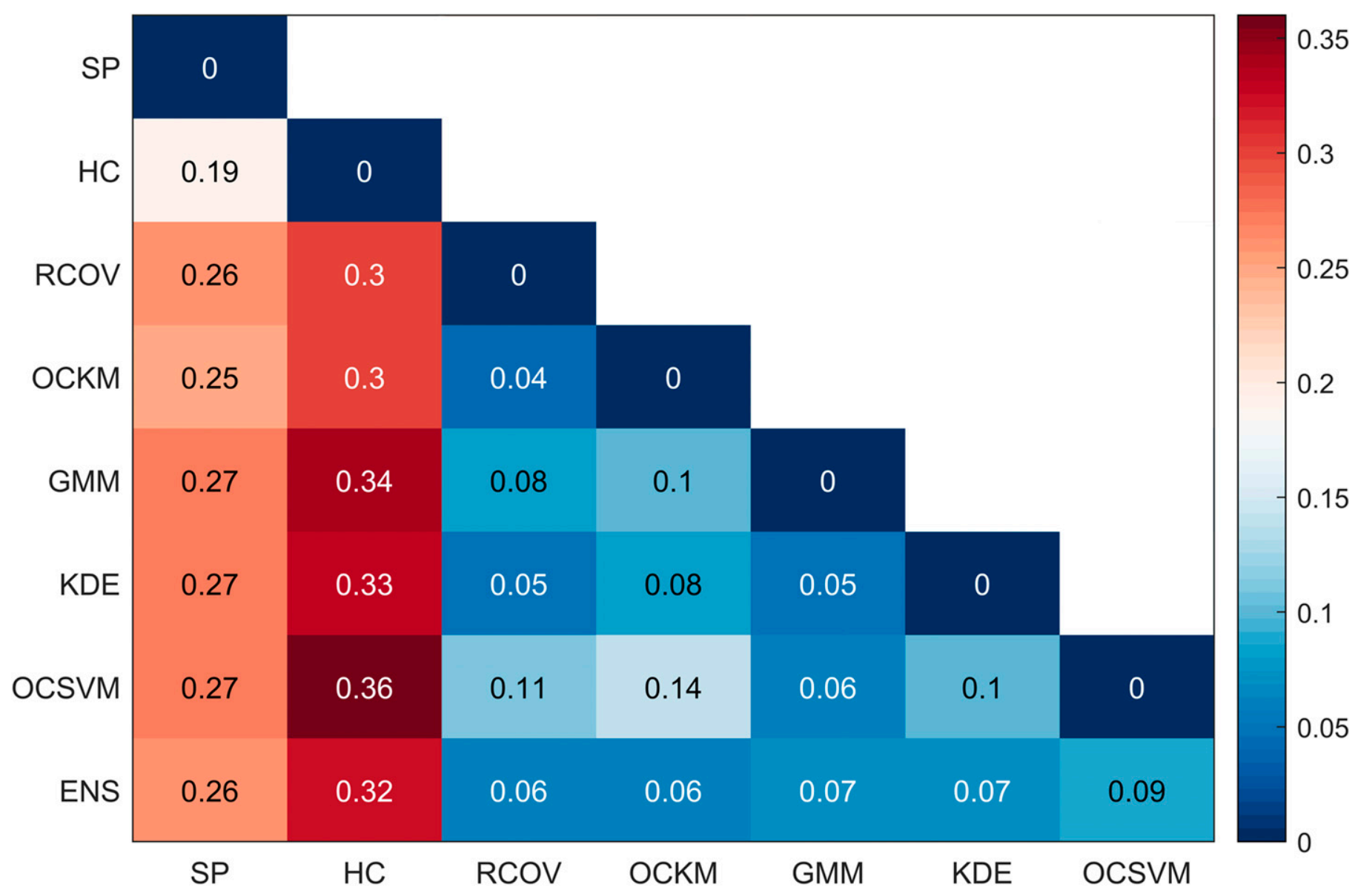

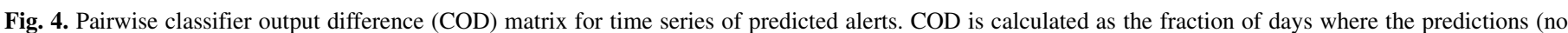

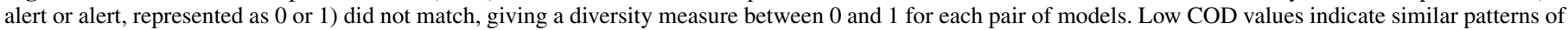

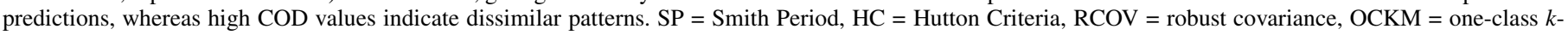

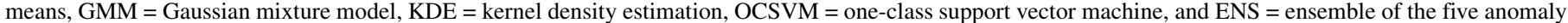
detection algorithms. 
(Wang et al. 2020). To my knowledge, this study represents a new application of anomaly detection techniques, including anomaly detection ensembles, to crop disease forecasting. All of the algorithms considered can be used with higher-dimensional data (more predictor variables) and mixtures of data types (e.g., categorical, continuous), and they are relatively robust to the assumptions that underlie traditional statistical procedures (e.g., normality, linearity, independence, homoscedasticity, etc.). The anomaly detection algorithms are also purely data driven, meaning they do not require any domain or specialist knowledge regarding the pathogen or disease nor any formalization of relationships between variables in the form of mathematical equations. In fact, the application and operation of algorithms in ML are sometimes described as a "black box" in that they typically do not explain how each input contributes to model output in a way that humans can understand (Rudin 2019). Consequently, correlation among predictor variables, which can affect coefficient estimates, is not a concern in the current context because the primary interest is in the predictive power of the algorithms and not model interpretability. The ML literature is also replete with many "feature selection algorithms" that can reduce the dimensionality of input data to the most important features for prediction if this is not known beforehand (Cai et al. 2018; Li et al. 2017; Miao and Niu 2016; Sheikhpour et al. 2017). Many of these methods are implemented within numerical computing environments such as MATLAB for easy integration with available ML algorithms. The techniques outlined in this study therefore offer significant advantages over conventional approaches to the derivation of plant disease prediction models. A relevant concern, however, in plant disease epidemiology is a lack of sufficient data for learning (i.e., small datasets). Many ML algorithms are "data hungry" and "overfitting" of the algorithm to training data is more likely in studies based on small sample sizes. Overfitting decreases the ability to generalize to unseen data, but this can typically be overcome by using lowercomplexity models (fewer weights, parameters etc.). Resampling techniques, such as the $k$-fold cross-validation procedure described in this study, can also be used to maximize the size of the learning dataset and check the stability of estimation performance across subsets of the data. All of the anomaly detection algorithms used here performed equally well in terms of predictive accuracy and stability during cross-validation (Table 1), and all are relatively easy to implement. If a single algorithm is to be recommended based on this study, then a critical challenge in deriving tools for forecasting risk of crop disease is consistency in performance across different geographic regions. The standard deviation of accuracy across Great Britain administrative regions was 2.6, 2.2, 4.4, 2.5, and 4.4\% for RCOV, OCKM, GMM, KDE, and OCSVM, respectively (Fig. 6 ), suggesting OCKM as the preferred algorithm on this basis. The app provided for download in Supplementary File S1 enables the interested reader to train and test their own crop disease forecasting tools based on any of the anomaly detection algorithms used in this study.

It is useful to discuss how the results and general approach of this study fit into the broader context of plant disease prediction. There are numerous ways to organize a taxonomy of plant disease prediction models, but most treatments of this topic categorize the literature based on modeling approach (De Wolf and Isard 2007; Krause and Massie 1975). In general, mechanistic, empirical, and ML approaches can be distinguished, although in practice hybrid approaches are occasionally utilized (van Maanen and Xu 2003). Models derived using a mechanistic or knowledge-based approach are often based on the results of experiments designed to investigate the pathosystem under controlled conditions (De Wolf and Isard 2007). Coupled ordinary and partial differential equations as well as

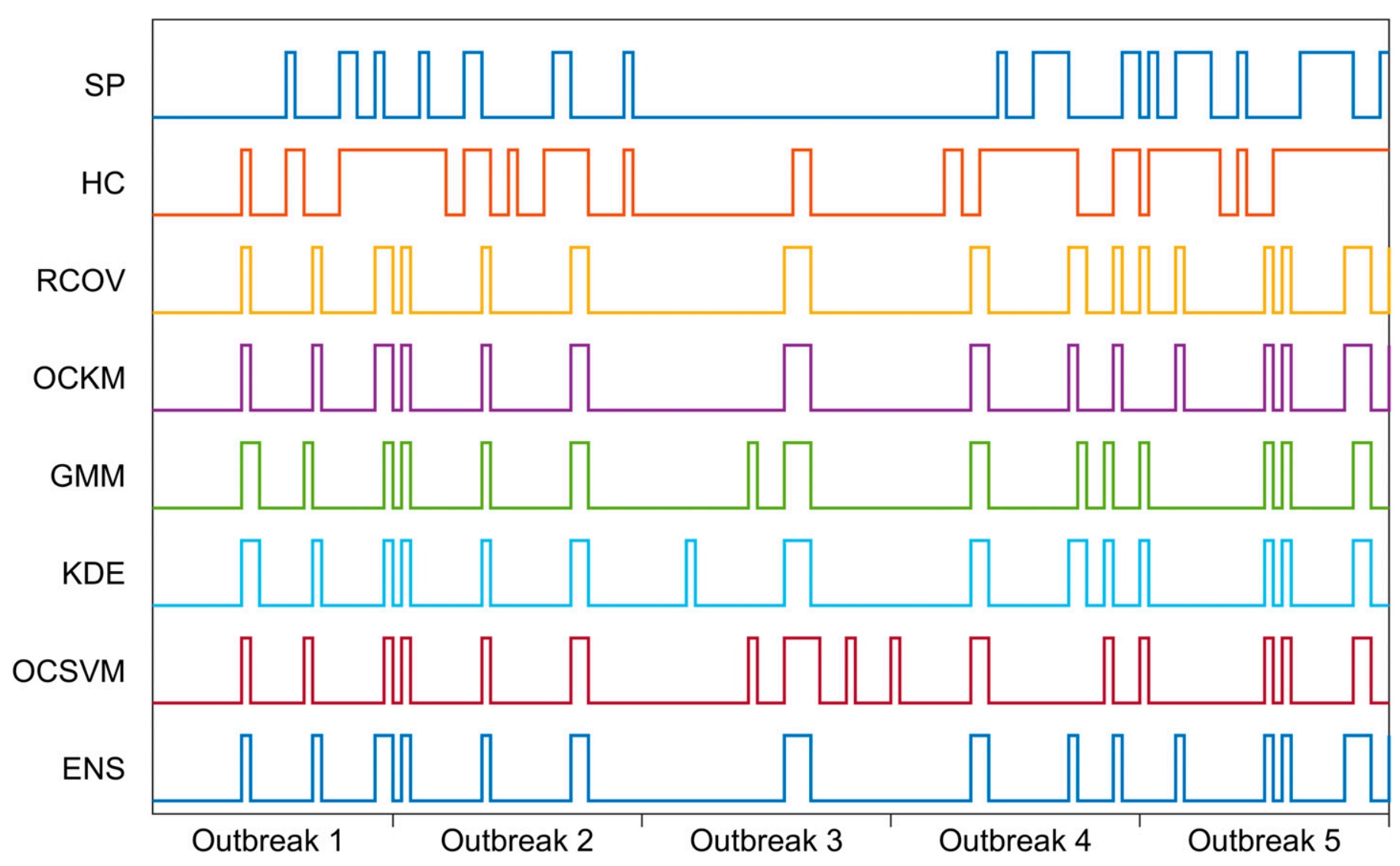

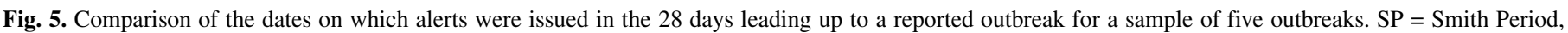

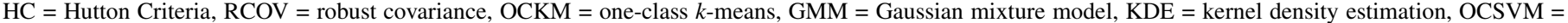
one-class support vector machine, and ENS = ensemble of the five anomaly detection algorithms. 
simpler curve-fitting procedures are especially common techniques (Madden et al. 2007). Mechanistic models are generally considered to have more explanatory capacity than purely empirical approaches and are therefore sometimes referred to as "whitebox" models. In contrast, empirical or correlative approaches are developed to describe a dataset with little concern about biological mechanisms, and they are often derived using field experiments that simulate production conditions (Madden and Campbell 1990). Traditionally, multivariate regression techniques were used, but advances in statistics, including linear and nonlinear mixed models, are allowing a more appropriate matching of data type and experimental (or survey) design to the statistical model used for analysis, in order to meet the objectives of the investigator (Madden 2006). Empirical models do not characterize cause-and-effect relationships within a disease system but can be used to make inferences about the underlying biology of a system (De Wolf and Isard 2007). In the ML approach, specialized algorithms are used to automate the model building process and iteratively learn from data to gain insights without explicit programming. These algorithms can be applied to any type of data and do not characterize causal relationships, nor are they used to make inferences about the underlying biology; ML is focused on results and the algorithms are designed to maximize predictive accuracy on unseen data. Although anomaly detection can be considered a subfield or specialization of ML, this work provides a new approach to plant disease prediction. Whereas the aforementioned approaches seek to quantify relationships in epidemiological data, anomaly detection algorithms are designed to identify data points that do not conform to other items present in a dataset. Each data point is assigned an anomaly score describing its outlier tendency, and in contrast to the conventional usage of such algorithms to identify outliers, in this study the least anomalous data points are used to define a decision boundary for triggering a disease risk alert (Fig. 2). Note that the decision threshold of the anomaly detection algorithms can be tuned to produce narrower or broader decision boundaries and thus any alert frequency desired, although there is a trade-off with forecast accuracy that would need to be considered with respect to the pathosystem of interest.

PLB provides a highly relevant case study to validate a new approach to plant disease prediction. A seemingly infinite number of host-pathogen-environment interactions can be used to quantify late blight risk, and this is reflected in the variety of forecasting tools that are currently deployed in different zones of potato production around the world. These range from simple models that consider disease development in the crop (Hyre 1954; Ullrich and Schrödter 1966; Wallin 1951), to integrated models that predict blight occurrence and favorable days for fungicide application (Hansen et al. 1995; Krause et al. 1975), to DSSs that integrate and organize information on the disease cycle, weather, plant growth, cultivar resistance, and disease pressure to make recommendations on the choice and timing of crop protection products (Skelsey 2008). For example, in Europe there are approximately 20 different DSSs currently deployed in industry for late blight management (Schepers et al. 2019). Although these systems are numerous and diverse in complexity, they typically all include a similar core set of temperature and humidity rules to quantify risk of infection. These rules are ordinarily derived from experimental data in the country of origin using isolates from the contemporary pathogen population and representative host varieties. This approach is problematic, however, when we consider that the predominant varieties grown, pathogen population structure, and climate can all vary widely within a country, which can lead to disparities in predictive accuracy among regions ( $\mathrm{Hu}$ et al. 2015). Lack of uniformity in SP performance among the potato growing regions of Great Britain

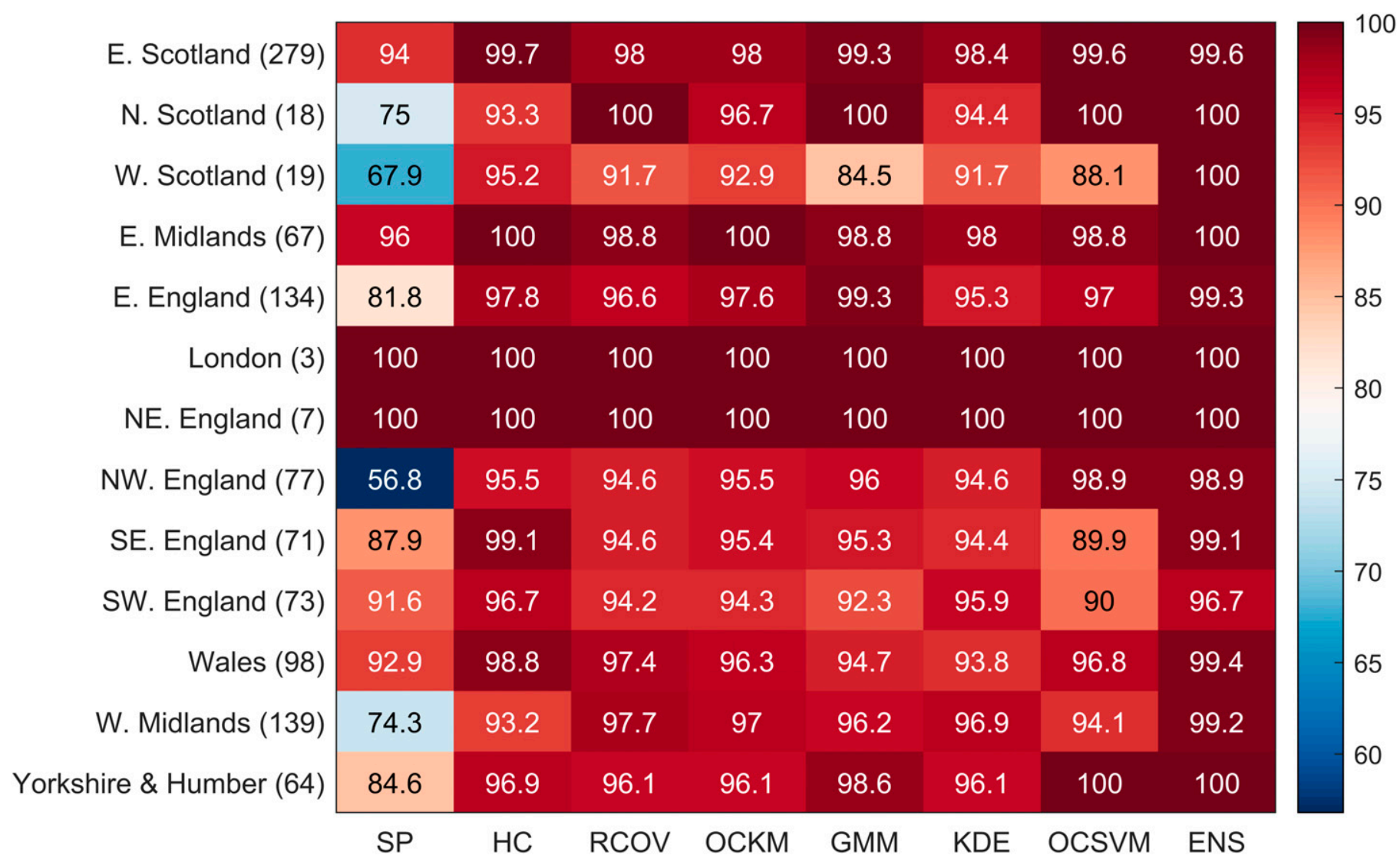

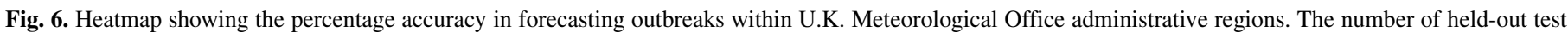

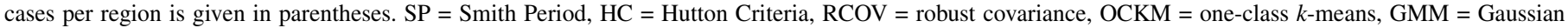

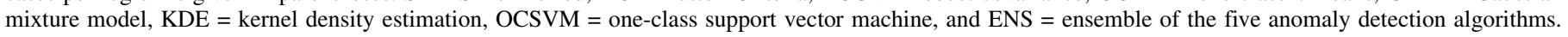


was one of the main issues raised by the Great Britain potato industry that prompted the development of the $\mathrm{HC}$ model as the new national warning system. The standard deviation of accuracy across Great Britain administrative regions was $13.2 \%$ for SP but only $6.8 \%$ for HC (Fig. 6). This issue has therefore been markedly improved in the current national warning system. Notably, the standard deviation of accuracy across regions was $0.9 \%$ for the multimodel ensemble, ENS. This result suggests a highly pragmatic solution to the potential problem of spatial variation in the performance of crop disease warning systems. Rather than adopt a "one-size-fits-all" approach whereby the same model is applied across large geographic areas, a multimodel ensemble can be implemented where the predictions of individual component models are combined to produce more accurate and robust region-specific alerts. It is generally agreed that the diversity of component models is a key ingredient for increasing the ensemble's accuracy (Zenobi and Cunningham 2001). This diversity manifests itself as disagreement or ambiguity among the ensemble members; thus, it is desirable that the predictions made by individual component models have low correlation. This was not the case in the current study, as the anomaly detection algorithms were all trained using the same predictor variables and subsets of the data, leading to similar decision boundaries and levels of predictive accuracy. Nevertheless, the presidential voting scheme used here, in which the most accurate model determined the ensemble result, was used to good effect to increase consistency in predictive performance across geographic regions and among the months of the growing season, which are two highly desirable traits for a warning system. As more data become available each year, the anomaly detection algorithms can be retrained and retested, their regional accuracy can be continuously evaluated, and the specifics of the ensemble voting strategy can be updated accordingly. Alternatively, the wide diversity of late blight forecasting systems currently deployed across Europe (Schepers et al. 2019) could also be combined to produce a European ensemble that takes advantage of the strengths and weaknesses of its component models to produce more accurate and robust forecasts of late blight risk within individual countries or climatic regions.

A fundamental challenge in deriving models for forecasting the risk of crop disease from surveys as opposed to experimental data is observation bias (Madden et al. 2007; Zadoks and Schein 1979). This bias can have several components, including spatial coverage bias (where not all fields are exposed to sampling) and detection bias (where some infected fields go undetected). This is often because the survey is focused on objectives other than a complete census of all outbreaks of disease. For example, in the case of the FAB PLB outbreak data used in this study, the objective is to sample the pathogen population in order to obtain information about population diversity, virulence, aggressiveness, and fungicide sensitivity that can be fed back to the potato industry. Sampling may be more intensive at the beginning of the growing season and decrease once blight is very active or once a scout has already sampled the population in a specific geographic area. Crop surveillance data could therefore be biased by "imperfect" detection and it is possible that resultant models describe patterns in the difficulty or manner with which pests or pathogens are detected rather than real patterns in their abundance and occurrence. The spatial distribution of reported outbreaks in the FAB data are reasonably uniform across the potato growing areas of Great Britain, with some concentrated regions along the eastern seaboard and scattered throughout Wales and the South West (Fig. 1A). These areas of higher incidence coincide with the areas where potato

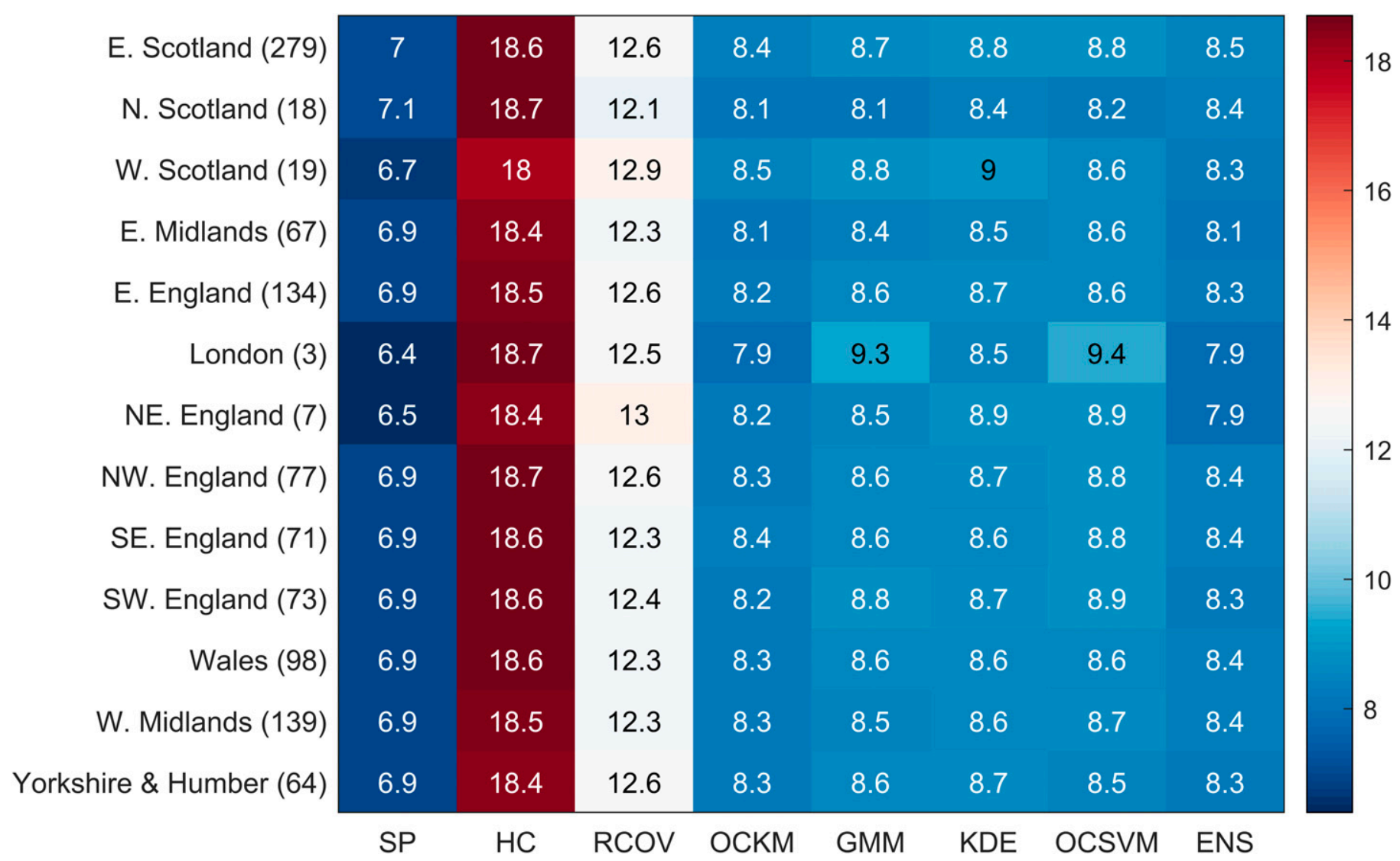

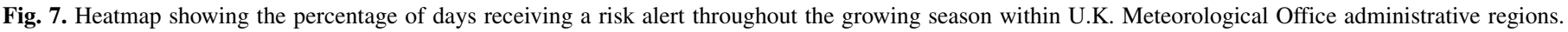

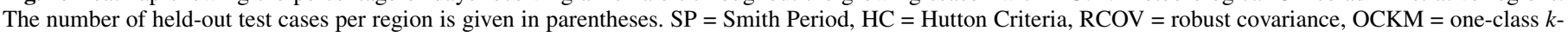

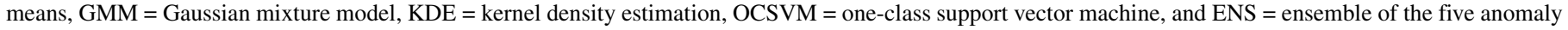
detection algorithms. 
production is most intense in Great Britain; therefore, the distribution of sampled outbreaks matches the distribution of potato quite well. The number of outbreaks reported in each country also mirrors the scale of potato production, which is greater in England, then Scotland and Wales, although incidence levels are relatively high for Scotland (Fig. 1B). In addition, the trend in outbreak reporting dates describes a typical epidemic curve for many crop diseases, where the number of new outbreaks increases to a peak then declines with the proportion of uninfected crops and a shift to less favorable climatic conditions (Fig. 1B). Taken together, this increases confidence that the quality and level of sampling activity is consistent across most of Great Britain and throughout the growing season. In addition, the trained anomaly detection algorithms generalized well to the "unseen" held-out test data and results were stable across test folds (Table 1), months of the growing season (Fig. 3), and different regions of Great Britain (Fig. 6); therefore, it is unlikely that observation bias affected the study results.

The application of anomaly detection techniques to data from a crop disease surveillance program enabled the development of a heterogeneous multimodel ensemble for forecasting crop disease risk. The ensemble has been demonstrated to be a simple but effective tool for forecasting outbreaks that provides flexibility in the tailoring of component models and/or their combination for specific geographic regions. The validity of the approach has been demonstrated via encouraging results from the hindcasting of historic outbreak events for a case study of PLB in Great Britain. Future development of the method introduced in this article will benefit from simulation studies to assess statistical biases in predictions (estimated anomalies) when there are problems with sampling coverage and detection bias and suggest improved methods for subsequent research. Experimental field trials with "live data" would also be beneficial for model validation, where the anomaly detection techniques or multimodel ensemble are used to forecast risk events and adapt spray regimes accordingly. The methodology developed in this study is extendable to other pests and pathogens and can be applied using standard weather forecast data.

\section{ACKNOWLEDGMENTS}

We thank AHDB Potatoes and the FAB scouts for providing the late blight outbreak data.

\section{LITERATURE CITED}

Aggarwal, C. C. 2013. Outlier analysis. Springer, Dordrecht, Netherlands.

Aggarwal, C. C., and Sathe, S. 2018. Outlier ensembles: An introduction. Springer International Publishing AG, Cham, Switzerland.

Arora, R., Singh, B., and Dhawan, A. K. 2012. Theory and practice of integrated pest management. Scientific Publishers, Jodhpur, India.

Benkeser, D., Ju, C., Lendle, S., and van der Laan, M. 2018. Online crossvalidation-based ensemble learning. Stat. Med. 37:249-260.

Boniecki, P., Koszela, K., Piekarska-Boniecka, H., Weres, J., Zaborowicz, M., Kujawa, S., Majewski, A., and Raba, B. 2015. Neural identification of selected apple pests. Comput. Electron. Agric. 110:9-16.

Brieman, L. 1996. Bagging predictors. Mach. Learn 24:123-140.

Cai, J., Luo, J., Wang, S., and Yang, S. 2018. Feature selection in machine learning: A new perspective. Neurocomputing 300:70-79.

Chen, G., Wang, H., and Ma, Z. 2006. Forecasting wheat stripe rust by discrimination analysis. Plant Prot. 32:24-27.

Clarke, B. 2004. Comparing Bayes model averaging and stacking when model approximation error cannot be ignored. J. Mach. Learn. Res. 4:683-712.

De Wolf, E., and Isard, S. A. 2007. Disease cycle approach to plant disease prediction. Annu. Rev. Phytopathol. 45:203-220.

De Wolf, E. D., and Franel, L. J. 1997. Neural networks that distinguish infection periods of wheat tan spot in an outdoor environment. Phytopathology 87:83-87.

Delerce, S., Dorado, H., Grillon, A., Rebolledo, M. C., Prager, S. D., Patiño, V. H., Garcés Varón, G., and Jiménez, D. 2016. Assessing weather-yield relationships in rice at local scale using data mining approaches. PLoS One 11:e0161620.

Désir, C., Bernard, S., Petitjean, C., and Heutte, L. 2013. One class random forests. Pattern Recognit. 46:3490-3506.
Domingos, P. 2000. Bayesian averaging of classifiers and the overfitting problem. ICML Conf. Proc. 747:223-230.

Douglas, P., Lau, E., Anderson, A., Kerr, W., Head, A., Wollner, M., Moyer, D., Durnhofer, M., Li, W., Bramen, J., and Cohen, M. 2013. Single trial decoding of belief decision making from EEG and fMRI data using independent components features. Front. Hum. Neurosci. 7:392.

Ferentinos, K. P. 2018. Deep learning models for plant disease detection and diagnosis. Comput. Electron. Agric. 145:311-318.

Freund, Y., and Schapire, R. E. 1997. A decision-theoretic generalization of on-line learning. JCoSS 55:119-139.

Ghosal, S., Blystone, D., Singh, A. K., Ganapathysubramanian, B., Singh, A., and Sarkar, S. 2018. An explainable deep machine vision framework for plant stress phenotyping. Proc. Natl. Acad. Sci. 115:4613-4618.

Goldstein, M., and Uchida, S. 2016. A comparative evaluation of unsupervised anomaly detection algorithms for multivariate data. PLoS One 11:e0152173.

Gonzalez-Sanchez, A., Frausto-Solis, J., and Ojeda-Bustamante, W. 2014. Predictive ability of machine learning methods for massive crop yield prediction. Span. J. Agric. Res. 12:313-328.

Guo, W., Fukatsu, T., and Ninomiya, S. 2015. Automated characterization of flowering dynamics in rice using field-acquired time-series RGB images. Plant Methods 11:7.

Guo, W., Rage, U. K., and Ninomiya, S. 2013. Illumination invariant segmentation of vegetation for time series wheat images based on decision tree model. Comput. Electron. Agric. 96:58-66.

Hamer, W. B., Birr, T., Verreet, J. A., Duttmann, R., and Klink, H. 2020. Spatio-temporal prediction of the epidemic spread of dangerous pathogens using machine learning methods. ISPRS Int. J. Geoinf. 9:44-62.

Hansen, J. G., Andersson, B., and Hermansen, A. 1995. NEGFRY: A system for scheduling chemical control of late blight in potatoes. Pages 201-208 in: Phytophthora infestans 150. L. J. Dowley, L. R. Cooke, T. Keane, and E. O'Sullivan, eds. European Association for Potato Research and Boole Press, Dublin, Ireland.

Hsu, C.-W., Chang, C.-C., and Lin, C.-J. 2001. A practical guide to support vector classification. https://www.csie.ntu.edu.tw/ cjlin/papers/guide/guide. pdf

Hu, X., Madden, L. V., Edwards, S., and Xu, X. 2015. Combining models is more likely to give better predictions than single models. Phytopathology 105:1174-1182.

Huang, S., Qi, L., Ma, X., Xue, K., Wang, W., and Zhu, X. 2015. Hyperspectral image analysis based on BoSW model for rice panicle blast grading. Comput. Electron. Agric. 118:167-178.

Hyre, R. A. 1954. Progress in forecasting late blight of potato and tomato. Plant Dis. Rep. 38:245-253.

Ishak, A. J., Hussain, A., and Mustafa, M. M. 2009. Weed image classification using Gabor wavelet and gradient field distribution. Comput. Electron. Agric. 66:53-61

Jain, A. K. 2010. Data clustering: 50 years beyond K-means. Pattern Recognit. Lett. 31:651-666.

Jain, A. K., and Dubes, R. C. 1988. Algorithms for clustering data. PrenticeHall, Upper Saddle River, NJ.

Japkowicz, N., and Shah, M. 2011. Evaluating learning algorithms: A classification perspective. Cambridge University Press, Cambridge, UK.

Kaundal, R., Kapoor, A. S., and Raghava, G. P. S. 2006. Machine learning techniques in disease forecasting: A case study on rice blast prediction. BMC Bioinformatics 7:485

Kaur, R., and Singh, S. 2016. A survey of data mining and social network analysis based anomaly detection techniques. Egypt. Inf. J. 17:199-216.

Khan, S. S., and Madden, M. G. 2010. A survey of recent trends in one class classification. Pages 188-197 in: Artificial intelligence and cognitive science. L. Coyle and J. Freyne, eds. Springer, Berlin, Germany.

Knauer, U., von Rekowski, C. S., Stecklina, M., Krokotsch, T., Pham Minh, T., Hauffe, V., Kilias, D., Ehrhardt, I., Sagischewski, H., Chmara, S., and Seiffert, U. 2019. Tree species classification based on hybrid ensembles of a convolutional neural network $(\mathrm{CNN})$ and random forest classifiers. Remote Sens. 11:2788.

Krause, R. A., and Massie, L. B. 1975. Predictive systems: Modern approaches to disease control. Annu. Rev. Phytopathol. 13:31-47.

Krause, R. A., Massie, L. B., and Hyre, R. A. 1975. BLITECAST, a computerized forecast of potato late blight. Plant Dis. Rep. 59:95-98.

Krawczyk, B., Minku, L. L., Gama, J., Stefanowski, J., and Woźniak, M. 2017. Ensemble learning for data stream analysis: A survey. Inf. Fusion 37:132-156.

Krstajic, D., Buturovic, L. J., Leahy, D. E., and Thomas, S. 2014. Crossvalidation pitfalls when selecting and assessing regression and classification models. J. Cheminform. 6:10.

Kurtulmus, F., Lee, W. S., and Vardar, A. 2014. Immature peach detection in colour images acquired in natural illumination conditions using statistical classifiers and neural network. Precis. Agric. 15:57-79.

Landschoot, S., Waegeman, W., Audenaert, K., Van Damme, P., Vandepitte, J., De Baets, B., and Haesaert, G. 2013. A field-specific web tool for the prediction of Fusarium head blight and deoxynivalenol content in Belgium. Comput. Electron. Agric. 93:140-148. 
Lantz, B. 2015. Machine learning with R. Packt Publishing, Birmingham, UK. Li, J., Cheng, K., Wang, S., Morstatter, F., Trevino, R. P., Tang, J., and Liu, H. 2017. Feature selection: A data perspective. ACM Comput. Surv. 50:94.

Liakos, K. G., Busato, P., Moshou, D., Pearson, S., and Bochtis, D. 2018. Machine learning in agriculture: A review. Sensors (Basel) 18:2674.

Lindsay, B. G. 1995. Mixture models: Theory, geometry and applications. NSF-CBMS Reg. Conf. Ser. Probab. Stat. 5:i-163.

Linker, R., Cohen, O., and Naor, A. 2012. Determination of the number of green apples in RGB images recorded in orchards. Comput. Electron. Agric. 81:45-57.

Lloyd, S. 1982. Least squares quantization in PCM. IEEE Trans. Inf. Theory 28:129-137.

Luo, H., Wang, D., Yue, C., Liu, Y., and Guo, H. 2018. Research and application of a novel hybrid decomposition-ensemble learning paradigm with error correction for daily PM10 forecasting. AtmRe 201:34-45.

Ma, J., Li, X., Wen, H., Fu, Z., and Zhang, L. 2015. A key frame extraction method for processing greenhouse vegetables production monitoring video. Comput. Electron. Agric. 111:92-102.

Madasamy, K., and Ramaswami, M. 2017. Data imbalance and classifiers: Impact and solutions from a big data perspective. Int. J. Comput. Intell. Res. 13:2267-2281.

Madden, L. V. 2006. Botanical epidemiology: Some key advances and its continuing role in disease management. Eur. J. Plant Pathol. 115:3-23.

Madden, L. V., and Campbell, C. L. 1990. Non-linear disease progress curves. Pages 181-229 in: Epidemics of plant diseases: Mathematical analysis and modeling. J. Kranz, ed. Springer, Berlin, Germany.

Madden, L. V. A., Hughes, G. A., and van den Bosch, F. 2007. The study of plant disease epidemics. American Phytopathological Society, St. Paul, MN

Mahalanobis, P. C. 1936. On the generalised distance in statistics. Proc. Natl. Inst. Sci. India 2:49-55.

Marko, O., Brdar, S., Panić, M., Šašić, I., Despotović, D., Knežević, M., and Crnojević, V. 2017. Portfolio optimization for seed selection in diverse weather scenarios. PLoS One 12:e0184198.

Maronna, R., Martin, R. D., and Yohai, V. J. 2006. Robust statistics: Theory and methods. Wiley, New York, NY.

Mazhelis, O. 2006. One-class classifiers: A review and analysis of suitability in the context of mobile-masquerader detection. S. Afr. Comput. J. 36: $29-48$.

Miao, J., and Niu, L. 2016. A survey on feature selection. Procedia Comput. Sci. 91:919-926

Misaki, M., Kim, Y., Bandettini, P. A., and Kriegeskorte, N. 2010. Comparison of multivariate classifiers and response normalizations for patterninformation fMRI. Neuroimage 53:103-118.

Mutka, A. M., and Bart, R. S. 2015. Image-based phenotyping of plant disease symptoms. Front. Plant Sci. 5:734.

Peterson, A. H., and Martinez, T. 2005. Estimating the potential for combining learning models. Pages 68-75 in: Proceedings of the ICML Workshop on Meta-Learning Association for Computing Machinery. International Conference on Machine Learning, Bonn, Germany.

Rahman, M., Blackwell, B., Banerjee, N., and Saraswat, D. 2015. Smartphonebased hierarchical crowdsourcing for weed identification. Comput. Electron. Agric. 113:14-23.

Rahnemoonfar, M., and Sheppard, C. 2017. Deep count: Fruit counting based on deep simulated learning. Sensors (Basel) 17:905.

Rocci, R., Gattone, S. A., and Di Mari, R. 2018. A data driven equivariant approach to constrained Gaussian mixture modeling. Adv. Data Anal. Classif. 12:235-260.

Rousseeuw, P. 1985. Multivariate estimation with high breakdown point. Pages 283-297 in: Mathematical statistics and applications. W. Grossmann, G. Pflug, I. Vincze, and W. Wertz, eds. Vol. B. Reidel, Dordrecht, Netherlands.

Rousseeuw, P. J. 1984. Least median of squares regression. J. Am. Stat. Assoc. 79:871-880.

Rousseeuw, P. J., and Van Driessen, K. 1999. A fast algorithm for the minimum covariance determinant estimator. Technometrics 41:212-223.

Rudin, C. 2019. Stop explaining black box machine learning models for high stakes decisions and use interpretable models instead. Nat. Mach. Intell. 1: 206-215.

Sa, I., Ge, Z., Dayoub, F., Upcroft, B., Perez, T., and McCool, C. 2016. DeepFruits: A fruit detection system using deep neural networks. Sensors (Basel) 16:1222.

Samuel, A. L. 1959. Some studies in machine learning using the game of checkers. IBM J. Res. Develop. 3:210-229

Schepers, H. T. A. M., Hausladen, H., Hansen, J. G., Abuley, I., Andersson, B., Liljeroth, E., Edin, E., Bain, R., Kennedy, C., Ritchie, F., Gaucher, D., Bugiani, R., Ivanovic, Ž., Hermeziu, M., Kildea, S., Filippov, A., Kuznetzova, M., Hannukkala, A., Eikemo, H., Osowski, J., Puidet, B., Kiiker, R., Musa, T., Sullam, K., Ronis, A., Vogelaar, K., and Vanhaverbeke, P. 2019. Epidemics and control of early and late blight, 2017 and 2018 in Europe. In: Proceedings of the Seventeenth EuroBlight Workshop. WUR Special Report 19, York, United Kingdom. H. T. A. M. Schepers, ed. Wageningen University and Research, Wageningen, Netherlands.

Schölkopf, B., Platt, J. C., Shawe-Taylor, J., Smola, A. J., and Williamson, R. C. 2001. Estimating the support of a high-dimensional distribution. Neural Comput. 13:1443-1471.

Schölkopf, B., and Smola, A. J. 2001. Learning with kernels: Support vector machines, regularization, optimization, and beyond. MIT Press, Cambridge, MA.

Sheikhpour, R., Sarram, M. A., Gharaghani, S., and Chahooki, M. A. Z. 2017. A survey on semi-supervised feature selection methods. Pattern Recognit. 64:141-158.

Silva, F. L., Grassi Sella, M. L., Francoy, T. M., and Costa, A. H. R. 2015. Evaluating classification and feature selection techniques for honeybee subspecies identification using wing images. Comput. Electron. Agric. 114: 68-77.

Silverman, B. W. 2018. Page 176 in: Density estimation for statistics and data analysis. Routledge, New York, NY.

Skelsey, P. 2008. Multi-scale modelling of potato late blight epidemics. Wageningen University, Wageningen, Netherlands.

Skelsey, P., Rossing, W. A. H., Kessel, G. J. T., and van der Werf, W. 2010. Invasion of Phytophthora infestans at the landscape level: How do spatial scale and weather modulate the consequences of spatial heterogeneity in host resistance? Phytopathology 100:1146-1161.

Sladojevic, S., Arsenovic, M., Anderla, A., Culibrk, D., and Stefanovic, D. 2016. Deep neural networks based recognition of plant diseases by leaf image classification. Comput. Intell. Neurosci. 2016:3289801.

Smith, L. P. 1956. Potato late blight forecasting by 90 per cent humidity criteria. Plant Pathol. 5:83-87.

Soh, Y., Hae, Y., Mehmood, A., Ashraf, R. H., and Kim, I. 2013. Performance evaluation of various functions for kernel density estimation. Open J. Appl. Sci. 3:58-64.

Su, L., Ma, L., Qin, N., Huang, D., and Kemp, A. 2018. Fault diagnosis of high-speed train bogie based on spectrogram and multi-channel voting. Pages 22-26 in: 2018 IEEE 7th Data Driven Control and Learning Systems Conference (DDCLS). IEEE, Washington, DC.

Su, Y. X., Xu, H., and Yan, L. J. 2017. Support vector machine-based open crop model (SBOCM): Case of rice production in China. Saudi J. Biol. Sci. 24:537-547.

Tax, D. M. J. 2001. One-class classification. Delft University of Technology, Delft, Netherlands.

Ubbens, J., Cieslak, M., Prusinkiewicz, P., and Stavness, I. 2018. The use of plant models in deep learning: An application to leaf counting in rosette plants. Plant Methods 14:6.

Ullrich, J., and Schrödter, H. 1966. Das problem der vorhersage des aufretens der kartoffelkrautfaule (Phytophthora infestans) und die moglichkeit seiner losung durch eine negativprognose. Nachrichtenblatt Dt. Pflanzenschutzd. Braunschw. 18:33-40.

van Maanen, A., and Xu, X. M. 2003. Modelling plant disease epidemics. Eur. J. Plant Pathol. 109:669-682.

Vapnik, V. N. 1995. The nature of statistical learning theory. Springer-Verlag, New York, NY.

Varma, S., and Simon, R. 2006. Bias in error estimation when using crossvalidation for model selection. BMC Bioinformatics 7:91.

Venugoban, K., and Ramanan, A. 2014. Image classification of paddy field insect pests using gradient-based features. Int. J. Mach. Learn. Comput. 4:1-5.

Wallin, J. R. 1951. Forecasting tomato and potato late blight in the northcentral region. Phytopathology 41:37.

Wang, X., Du, Y., Lin, S., Cui, P., Shen, Y., and Yang, Y. 2020. adVAE: A selfadversarial variational autoencoder with Gaussian anomaly prior knowledge for anomaly detection. Knowl. Base. Syst. 190:105187.

Wolpert, D. H. 2002. The supervised learning no-free-lunch theorems. Pages 25-42 in: Soft computing and industry: Recent applications. R. Roy, M. Köppen, S. Ovaska, T. Furuhashi, and F. Hoffmann, eds. Springer,London, UK.

Xu, W., Wang, Q., and Chen, R. 2018. Spatio-temporal prediction of crop disease severity for agricultural emergency management based on recurrent neural networks. GeoInformatica 22:363-381.

Yamamoto, K., Guo, W., Yoshioka, Y., and Ninomiya, S. 2014. On plant detection of intact tomato fruits using image analysis and machine learning methods. Sensors (Basel) 14:12191-12206.

Yamamoto, K., Togami, T., and Yamaguchi, N. 2017. Super-resolution of plant disease images for the acceleration of image-based phenotyping and vigor diagnosis in agriculture. Sensors (Basel) 17:2557.

Zadoks, J. C., and Schein, R. D. 1979. Epidemiology and plant disease management. Oxford University Press, New York, NY.

Zenobi, G., and Cunningham, P. 2001. Using diversity in preparing ensembles of classifiers based on different feature subsets to minimize generalization error. Pages 576-587 in: 2001 European Conference on Machine Learning. Lecture Notes in Computer Science. Vol. 2167. L. De Raedt and P. Flach, eds. Springer, Berlin, Germany. 